\title{
Tribes of cubic partial cubes
}

\author{
Sandi Klavžar $\|^{1 \mid}$ and Sergey Shpectorov" \\ ${ }^{1}$ Department of Mathematics and Computer Science, FNM, University of Maribor, Gosposvetska 84, 2000 Maribor, \\ Slovenia \\ ${ }^{2}$ School of Mathematics, University of Birmingham, Edgbaston, Birmingham B15 2TT, United Kingdom \\ received september 16, 2005, revised may 29, 2007, accepted october 15, 2007.
}

Partial cubes are graphs isometrically embeddable into hypercubes. Three infinite families and a few sporadic examples of cubic partial cubes are known. The concept of a tribe is introduced as means to systematize the known examples and establish relations among them. Efficient methods of computation of tribes are developed and several concrete tribes, that include known, as well as new cubic partial cubes, are computed by hand and with the use of a computer.

Keywords: partial cube; hypercube; isometric embedding; algorithm; tribe

\section{Introduction}

Graphs that admit isometric embeddings into hypercubes are known as partial cubes. They were introduced by Graham and Pollak (22) as a model for interconnection networks and were extensively studied afterwards, see, for instance, (1, 6, 13, 15, 18, 22, 30, 36, 37) and the books (12, 25). Partial cubes also found several other applications. They are used in mathematical chemistry (27, 28), computational biology $(5 ; 14)$, media theory (17), and psychology (34). The variety of partial cubes is surprisingly rich, a fact that can in particular be justified by the variety of median graphs. The latter graphs form a very special subclass of partial cubes (cf. (33)), yet it has been demonstrated in (26) that there are, roughly speaking, as many median graphs as triangle-free graphs. More precisely, it is proved that a given graph $G$ on $n$ vertices and $m$ edges is triangle-free if and only if its associated graph $\widetilde{G}$ is a median graph, where $\widetilde{G}$ is a graph on $n+m+1$ vertices and $n+2 m$ edges. A related result due to Bandelt and van de Vel (2) asserts that the simplex graph of an arbitrary graph is always median. Note however that the size of the simplex graph can be exponential with respect to the original graph.

It is therefore surprising that it is not easy to find regular partial cubes. This has first been encountered by Weichsel (35) who proved that among distance regular graphs only hypercubes, even cycles, and the middle-level graphs are partial cubes. Since the Cartesian product of (regular) partial cubes is again a

\footnotetext{
${ }^{\dagger}$ Supported in part by the Ministry of Science of Slovenia under the grant P1-0297. The author is also with the Institute of Mathematics, Physics and Mechanics, Jadranska 19, 1000 Ljubljana, Slovenia.

‡Research partly supported by the Ministry of Science of Slovenia. 
(regular) partial cube, there are of course several infinite series of regular partial cubes. For instance, as even cycles are partial cubes, so are the toroidal graphs $C_{2 k} \square C_{2 n}, k, n \geq 2$.

The problem of classifying cubic partial cubes is around for about a decade. As far as we know, the problem has been explicitly written down for the first time in (29), where it is proved among other statements that $P(10,3)$ is the only partial cube among the generalized Petersen graphs. Some other examples of cubic partial cubes were known before, cf. (11), while in (3) and in (4) several new examples were constructed. It has been recently proved that among the cubic plane graphs whose faces are squares and hexagons there are precisely 5 partial cubes, all of them known before, see (9).

Clearly, even prisms $K_{2} \square C_{2 n}$ form an infinite series of cubic partial cubes. Besides this series, only a few sporadic examples have been known until recently ${ }^{(i)}$, cf. Table 1 . In the present paper we continue the search for cubic partial cubes and for this sake we introduce tribes of partial cubes. Using this concept, the related theory, and computational methods, we determine the tribes of all previously known cubic partial cubes. Among the graphs in these tribes we find 21 new cubic partial cubes. The computations were performed partly by hand and partly in the computer algebra system GAP, see (19).

We proceed as follows. In the remainder of this section basic definitions are given. Then, in Section 2. we present the list of previously known cubic partial cubes. In the subsequent section we revisit the well-known concepts of expansion and contraction and in particular introduce superconvex partitions. In Section 4 our central concept-tribe-is introduced. Then we consider the tribe of the generalized Petersen graph $P(10,3)$ and show that it consists only of $P(10,3)$ itself. The remaining known cubic partial cubes are planar, hence in Section 6 we restrict our investigations to the planar case and prepare the necessary theory for our computations. We also show that the even prisms $K_{2} \square C_{2 n}$ form a tribe. Finally, in Section 7, we presents the results of the explicit computations of the tribes of the remaining graphs from Table 1

A subgraph $H$ of $G$ is called isometric if $d_{H}(u, v)=d_{G}(u, v)$ for all $u, v \in V(H)$, where $d_{G}(u, v)$ denotes the usual shortest path distance. Also, $H$ is convex, if for all $u, v \in V(H)$, all shortest $u, v$ paths from $G$ belong to $H$. A convex subgraph is isometric but the converse need not be true. We call a subgraph $H$ superconvex if for all $u, v \in H$, all paths between $u$ and $v$ of length $\leq d_{G}(u, v)+1$ are contained in $H$. In general superconvexity is a stronger property than convexity. For bipartite graphs, however, the two properties are equivalent. A graph $G$ is a median graph if there exists a unique vertex $x$ to every triple of vertices $u, v$, and $w$ such that $x$ lies simultaneously on a shortest $u, v$-path, shortest $u, w$-path, and shortest $w, v$-path.

The Cartesian product $G \square H$ of graphs $G$ and $H$ is the graph with vertex set $V(G) \times V(H)$ where the vertex $(a, x)$ is adjacent to the vertex $(b, y)$ whenever $a b \in E(G)$ and $x=y$, or $a=b$ and $x y \in E(H)$. The Cartesian product is commutative and associative, the product of $k$ copies of $K_{2}$ is the $k$-dimensional hypercube or $k$-cube $Q_{k}$. A graph is called prime (with respect to the Cartesian product) if it cannot be written as the Cartesian product of two nontrivial graphs. It has been observed in (3) that a cubic partial cube is either an even prism or it is a prime graph.

(i) In September 2005, when this paper was in preparation, David Eppstein informed us of his discovery of a connection between cubic partial cubes and simplicial arrangements of lines. This leads to two more infinite series and a number of new sporadic examples of cubic partial cubes, see (16). We briefly discuss this development in Section 8 


\section{Known cubic partial cubes}

In Table 1 the list of known sporadic cubic partial cubes is presented, where $n$ stands for the number of vertices and dim for the dimension of the smallest hypercube into which the graph embeds isometrically.

\begin{tabular}{|c|c|c|c|}
\hline$n$ & $\operatorname{dim}$ & graph & ref \\
\hline 20 & $\overline{5}$ & $\mathrm{P}(10,3)$ & $(\overline{(11 ; 29)}$ \\
\hline 24 & 6 & $B_{1}$ & $(9 ; 11)$ \\
\hline 30 & 7 & $B_{1}^{\prime}$ & (3) \\
\hline 32 & 7 & chamfered cube & $(9 ; 11)$ \\
\hline 32 & 7 & twisted chamfered cube & $(9 ; 11)$ \\
\hline 36 & 8 & $B_{1}^{\prime \prime}$ & (3) \\
\hline 42 & 9 & $B_{1}^{\prime \prime \prime}$ & (3) \\
\hline 48 & 8 & $\mathcal{C} \mathcal{I}\left(G_{1}\right)$ & (4) \\
\hline 48 & 9 & $\mathcal{C} \mathcal{I}\left(Q_{3}\right)$ & $(10 ; 11)$ \\
\hline 48 & 10 & $B_{2}$ & (3) \\
\hline 80 & 8 & $\mathcal{C} \mathcal{I}\left(G_{2}\right)$ & (4) \\
\hline 80 & 8 & $\mathcal{C} \mathcal{I}\left(G_{3}\right)$ & (4) \\
\hline 96 & 12 & $\mathcal{C I}\left(G_{4}\right)$ & (4) \\
\hline 96 & 13 & $\mathcal{C} \mathcal{I}\left(G_{5}\right)$ & (4) \\
\hline 120 & 15 & $\mathcal{C I}$ (Icosahedron) & $(10 ; 11)$ \\
\hline
\end{tabular}

Tab. 1: Known cubic partial cubes.

Let us have a closer look at the table. There we find two short series of graphs and 6 additional examples. The latter examples are the generalized Petersen graph $P(10,3)$, the truncated octahedron $B_{1}$, the chamfered cube, the twisted chamfered cube, the truncated cuboctahedron $\mathcal{C I}\left(Q_{3}\right)$, and the truncated icosidodecahedron $\mathcal{C} \mathcal{I}$ (Icosahedron). The truncated octahedron is also known as the permutahedron $\Pi_{3}$ and has appeared elsewhere, cf. (20).

The first short series $B_{1}, B_{1}^{\prime}, B_{1}^{\prime \prime}, B_{1}^{\prime \prime \prime}, B_{2}$ with 4 new examples has been constructed in (3) by means of expansion procedures. It follows from the investigations in the present paper that this series cannot be extended.

In further search for new cubic partial cubes the following operation turned out to be useful. Let $G$ be a graph without vertices of degree one embedded into some closed surface. Then the cubic inflation $\mathcal{C I}(G)$ is defined as follows, see (4). Replace each vertex $v \in V(G)$ of degree $d$ by a $2 d$-cycle $Q_{v}$, and then replace every edge $u v$ of $G$ by two edges joining $Q_{u}$ and $Q_{v}$ in such a way that an embedded cubic graph on the same surface is obtained in which all cycles $Q_{v}$ are facial and all edges of $G$ give rise to 4-faces in that embedding. $\mathcal{C I}(G)$ can also be described as the dual of the barycentric subdivision of $G(4,31)$ or the truncation of the medial graph of $G(21)$ that can in turn be described as the $\{0,1,2\}$-Wythoffian (7, 8). Note that the truncated octahedron $B_{1}$ can also be represented as $\mathcal{C} \mathcal{I}\left(K_{4}\right)$.

The graphs $G_{1}, \ldots, G_{5}$ that appear in Table 1 are shown in Fig. 11. The cubic inflations of these graphs provided the last series of five cubic partial cubes. We conclude this section by pointing out that it has been verified by a computer search that Table 1 is complete up to 30 vertices (3). In other words, there are just three prime cubic partial cubes on at most 30 vertices: $P(10,3), B_{1}$, and $B_{1}^{\prime}$. 

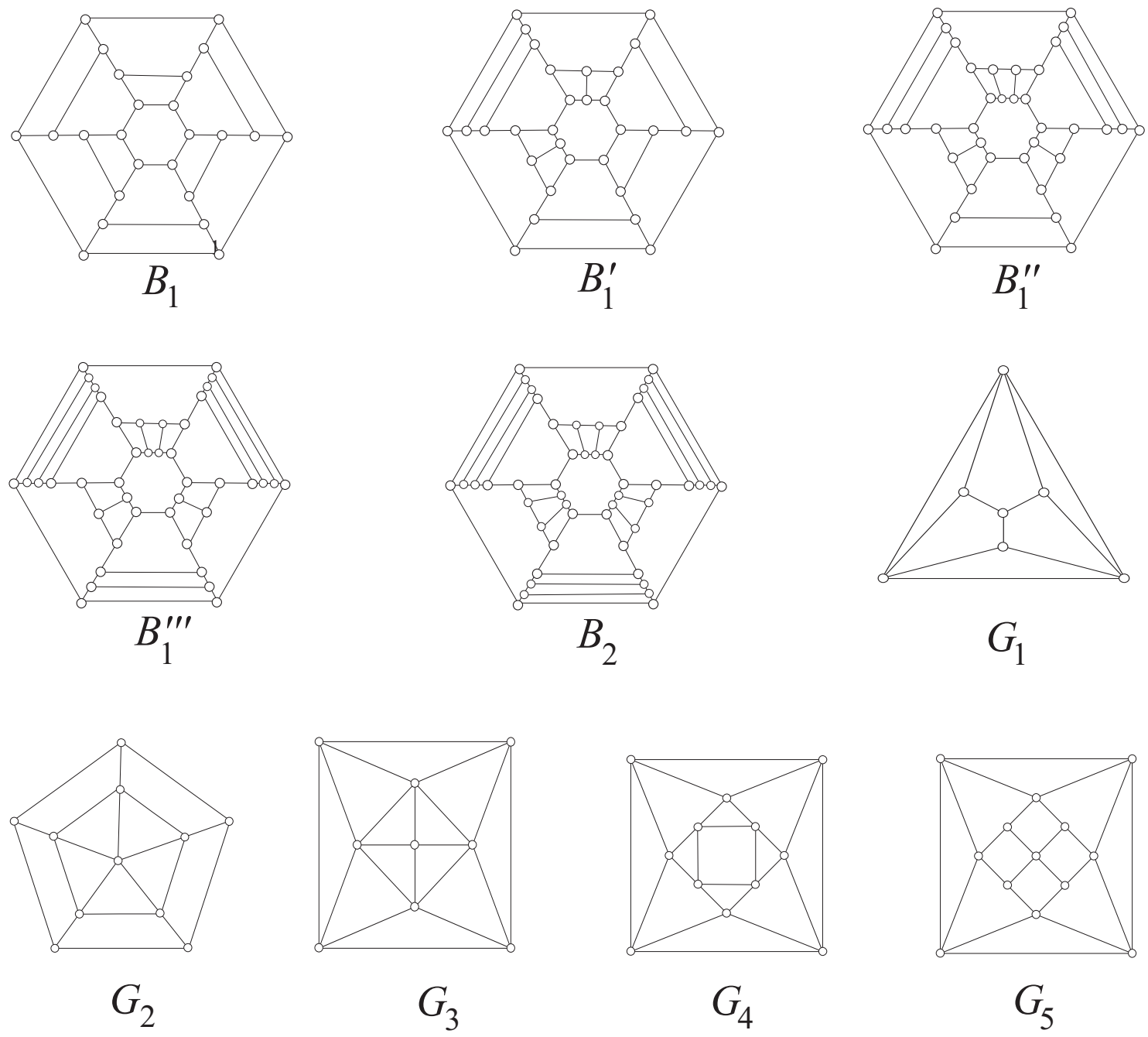

Fig. 1: Graphs from Table 1 


\section{Expansion and contraction}

The concept of expansion is due to Mulder (32, 33) in the context of median graphs and Chepoi (6) in the context of partial cubes (and partial Hamming graphs).

We say that two nonempty isometric subgraphs $H_{0}$ and $H_{1}$ form an isometric cover of a graph $H$ provided that $H=H_{0} \cup H_{1}$, by which we mean that $V(H)=V\left(H_{0}\right) \cup V\left(H_{1}\right)$ and $E(H)=E\left(H_{0}\right) \cup$ $E\left(H_{1}\right)$. If $H$ is connected then $H_{0} \cap H_{1} \neq \emptyset$ for every isometric cover $H_{0}, H_{1}$, provided that $H_{0}$ and $H_{1}$ are themselves nonempty.

Suppose $H_{0}, H_{1}$ is an isometric cover of $H$. For $i=0,1$, let $H_{i}^{\prime}$ be an isomorphic copy of $H_{i}$, and for a vertex $u \in H_{0} \cap H_{1}$, let $u_{i}$ be the corresponding vertex in $H_{i}^{\prime}$. The expansion of $H$ with respect to $H_{0}, H_{1}$ is the graph $G$ obtained from the disjoint union of $H_{0}^{\prime}$ and $H_{1}^{\prime}$, where for each $u \in H_{0} \cap H_{1}$ the vertices $u_{0}$ and $u_{1}$ are joined by a new edge in $G$. Chepoi (6) proved that a graph is a partial cube if and only if it can be obtained from $K_{1}$ by a sequence of expansions.

We next describe the operation of contraction. We say that superconvex subgraphs $G_{0}$ and $G_{1}$ form a superconvex partition of $G$ if $G_{0}$ and $G_{1}$ partition $G$. (More precisely, $V\left(G_{0}\right)$ and $V\left(G_{1}\right)$ partition $V(G)$.) When $G$ is partitioned by subgraphs $G_{0}, G_{1}$, we let $M\left(G_{0}, G_{1}\right)$ denote the set of edges of $G$ connecting $G_{0}$ with $G_{1}$. If $G$ is connected and $G_{0}, G_{1}$ are nonempty then $M\left(G_{0}, G_{1}\right)$ is also nonempty. For $e \in M\left(G_{0}, G_{1}\right)$, we let $e_{0}$ and $e_{1}$ denote the end vertices of $e$ that are in $G_{0}$ and $G_{1}$, respectively.

Proposition 3.1 Suppose subgraphs $G_{0}$ and $G_{1}$ partition $G$. Then $G_{0}, G_{1}$ is a superconvex partition if and only if $d_{G_{0}}\left(e_{0}, f_{0}\right)=d_{G_{1}}\left(e_{1}, f_{1}\right)$ for all $e, f \in M\left(G_{0}, G_{1}\right)$.

Proof: Suppose $G_{0}$ and $G_{1}$ are superconvex. In particular, for $i=0,1, d_{G_{i}}(u, v)=d_{G}(u, v)$ for all $u, v \in G_{i}$. Suppose by way of contradiction that, say, $d_{G}\left(e_{0}, f_{0}\right)<d_{G}\left(e_{1}, f_{1}\right)$. This means that the length of a shortest path from $e_{1}$ to $f_{1}$ passing through $e_{0}$ and $f_{0}$ is $d_{G}\left(e_{0}, f_{0}\right)+2 \leq d_{G}\left(e_{1}, f_{1}\right)+1$. Since $G_{1}$, is superconvex, we get $e_{0}, f_{0} \in G_{1}$, a contradiction. Hence, $d_{G}\left(e_{0}, f_{0}\right) \geq d_{G}\left(e_{1}, f_{1}\right)$, and we get equality by symmetry.

Now suppose $d_{G_{0}}\left(e_{0}, f_{0}\right)=d_{G_{1}}\left(e_{1}, f_{1}\right)$ for all $e, f \in M\left(G_{0}, G_{1}\right)$. Suppose by contradiction that, say, $G_{0}$ is not superconvex. Then, for some $u, v \in G_{0}$, there exists a path between $u$ and $v$ of length at most $d_{G}(u, v)+1$, that is not fully contained in $G_{0}$. Let $e$ be the first edge on this path, that crosses from $G_{0}$ to $G_{1}$, and let $f$ be the first edge on the path, crossing back in $G_{0}$. The part of the path between $e_{0}$ and $f_{0}$ has length at most $d_{G}\left(e_{0}, f_{0}\right)+1$. Furthermore, every intermediate vertex on this subpath lies in $G_{1}$. Thus, $d_{G}\left(e_{0}, f_{0}\right)+1 \geq d_{G_{1}}\left(e_{1}, f_{1}\right)+2$, that is, $d_{G}\left(e_{0}, f_{0}\right)>d_{G_{1}}\left(e_{1}, f_{1}\right)$. This yields a contradiction, since $d_{G_{0}}\left(e_{0}, f_{0}\right) \geq d_{G}\left(e_{0}, f_{0}\right)$.

Suppose $G_{0}$ and $G_{1}$ form a superconvex partition of $G$. The contraction of $G$ with respect to $G_{0}, G_{1}$ is the graph $H$ obtained from $G$ by removing from it all edges $e \in M\left(G_{0}, G_{1}\right)$ and by identifying $e_{0}$ with $e_{1}$ for each $e$. Notice that it follows from Proposition 3.1 that $e_{0}=f_{0}$ for $e, f \in M\left(G_{0}, G_{1}\right)$ implies $e_{1}=f_{1}$, and vice versa. If $e_{i}$ is adjacent with $f_{i}$ then also $e_{1-i}$ is adjacent with $f_{1-i}$. In this case, the edge $e_{0} f_{0}$ is identified with the edge $e_{1} f_{1}$.

The subgraphs $G_{0}, G_{1}$, and the set of edges $M\left(G_{0}, G_{1}\right)$ have already appeared in the literature on partial cubes. For an edge $a b$ of a graph $G$ let

$$
\begin{aligned}
& W_{a b}=\left\{u \mid u \in V(G), d_{G}(u, a)<d_{G}(u, b)\right\}, \\
& W_{b a}=\left\{u \mid u \in V(G), d_{G}(u, b)<d_{G}(u, a)\right\}, \\
& F_{a b}=\left\{u v \in E(G) \mid u \in U_{a b}, v \in U_{b a}\right\} .
\end{aligned}
$$


The first characterization of partial cubes is due to Djoković (13): A graph $G$ is a partial cube if and only if it is bipartite and if for every edge $a b$ of $G$ the subgraphs $W_{a b}$ and $W_{b a}$ are (super)convex.

Proposition 3.2 If $G_{0}, G_{1}$ is a superconvex partition of a graph $G$ then $G_{0}=W_{e_{0} e_{1}}, G_{1}=W_{e_{1} e_{0}}$, and $M\left(G_{0}, G_{1}\right)=F_{e_{0} e_{1}}$ for every $e \in M\left(G_{0}, G_{1}\right)$.

Proof: Set $a=e_{0}$ and $b=e_{1}$. It suffices to show that $G_{0} \subseteq W_{a b}$ and, symmetrically, $G_{1} \subseteq W_{b a}$. Suppose $d_{G}(u, b) \leq d_{G}(u, a)$ for some $u \in G_{0}$. Then there exists a path from $a$ to $u$ via $b$, that has length $d_{G}(u, b)+1 \leq d_{G}(u, a)+1$. By superconvexity of $G_{0}$, we get that $b \in G_{0}$, a contradiction.

We end this section with a remark that if a graph $G$ is obtained from a graph $H$ via expansion with respect to an isometric cover $H_{0}, H_{1}$ then the images of $H_{0}$ and $H_{1}$ in $G$ (that is, $H_{0}^{\prime}$ and $H_{1}^{\prime}$ ) form a superconvex partition of $G$. Consequently, $H$ can be recovered from $G$ via the corresponding contraction. Reversely, if a graph $H$ is obtained from a graph $G$ via contraction with respect to a superconvex partition $G_{0}, G_{1}$ then the images of $G_{0}$ and $G_{1}$ in $H$ form an isometric cover, and $G$ can be recovered from $H$ via the expansion with respect to this isometric cover. In this sense, expansion and contraction are inverse to each other.

\section{Tribes}

The graphs, on which we now focus our attention, are regular partial cubes, especially, cubic partial cubes. The class of partial cubes is closed with respect to expansions and contractions. This is, in essence, the result of (6). Let us now investigate conditions, under which expansion and contraction preserve regularity. The following two propositions are applicable to arbitrary regular graphs (not necessarily partial cubes).

Suppose $H$ is a regular graph of degree $n$. Suppose further that $G$ is an expansion of $H$ with respect to an isometric cover $H_{0}, H_{1}$. For $v \in H_{0} \cap H_{1}$ let $a_{v}$ be the number of neighbors of $v$ outside of $H_{0}, b_{v}$ the number of neighbors outside of $H_{1}$, and $c_{v}$ the number of neighbors in $H_{0} \cap H_{1}$.

Proposition 4.1 Under the above assumptions, $G$ is regular if and only if one of the following is true:

(1) $H_{0}=H_{1}=H$, and $G=H \square K_{2}$ is of degree $n+1$; or

(2) G has degree $n$ and, furthermore, $a_{v}=b_{v}=1$ and $c_{v}=n-2$ for all $v \in H_{0} \cap H_{1}$.

Proof: If $H_{0}=H_{1}=H$ then, clearly, (1) holds. Otherwise, there is a vertex outside of $H_{0} \cap H_{1}$. The corresponding vertex of $G$ has degree $n$. Therefore, if $G$ is regular, it must be of degree $n$.

Let $v \in H_{0} \cap H_{1}$. Clearly, $a_{v}+b_{v}+c_{v}=n$. Since the degree of $v_{0}$ in $G$ is $1+b_{v}+c_{v}$ and the degree of $v_{1}$ is $a_{v}+1+c_{v}$, we get the claim.

In the cubic case, $G$ is again cubic if and only if $a_{v}=b_{v}=c_{v}=1$. In particular, $H_{0} \cap H_{1}$ must be a disjoint union of edges. We will call such an expansion a cubic expansion.

Suppose now that $H$ is obtained from $G$ by contraction with respect to a superconvex partition $G_{0}, G_{1}$. For $i=0,1$, set $U_{i}=\left\{e_{i} \mid e \in M\left(G_{0}, G_{1}\right)\right\}$. According to Proposition 3.1, the mapping sending $e_{0}$ to $e_{1}$ for all $e \in M\left(G_{0}, G_{1}\right)$, establishes an isomorphism between distance spaces $U_{0}$ and $U_{1}$. In particular, this mapping is also an isomorphism of the graphs induced on $U_{0}$ and $U_{1}$.

Suppose $G$ is regular of degree $n$.

Proposition 4.2 The contraction $H$ is regular if and only if one of the following holds: 
(1) $U_{0}=G_{0}$ and $U_{1}=G_{1}$; in this case $H$ has degree $n-1$;

(2) the graph on $U_{0}$ is regular of degree $n-2$; here $H$ has degree $n$.

Proof: If $U_{0}=G_{0}$ and $U_{1}=G_{1}$ then, clearly, (1) holds. If there is a vertex in $G$ outside of $U_{0} \cup U_{1}$ then the corresponding vertex of $H$ has degree $n$. So $H$ must be of degree $n$. For $e \in M\left(G_{0}, G_{1}\right)$, let $a$ be the number of neighbors of $e_{0}$ in $G_{0} \backslash U_{0}$ and $b$ the number of neighbors in $U_{0}$. Then $n=a+b+1$. Since $e_{1}$ has the same number $b$ of neighbors in $U_{1}$, it follows that $e_{1}$ must have exactly $a$ neighbors in $G_{1} \backslash U_{1}$. Therefore, the unified $e_{0}, e_{1}$ vertex of $H$ has degree $a+b+a$. This yields $a=1$ and $b=n-2$.

When $G$ is cubic, the contraction $H$ is also cubic if and only if the graph on $U_{0}$ is a disjoint union of edges. In this case we call the contraction a cubic contraction.

We can now introduce our central concept - tribes. If $G$ is a cubic partial cube then every graph obtained from $G$ by cubic expansion or cubic contraction is again a cubic partial cube. A tribe of cubic partial cubes is a minimal nonempty collection of such graphs, closed under cubic expansions and cubic contractions. The tribe containing a particular graph $G$ will be referred to as the $G$-tribe.

\section{The case of $P(10,3)$}

In this section we prove the following

Proposition 5.1 The generalized Petersen graph $P(10,3)$ admits no cubic expansions and no cubic contractions. Consequently, the $P(10,3)$-tribe consists only of $P(10,3)$ itself.

We will prove this proposition in a sequence of lemmas, that also introduce some of the ideas involved in tribe computations.

Lemma 5.2 If the contraction of a cubic graph $G$ with respect to a superconvex partition $G_{0}, G_{1}$ is a cubic contraction then every edge from $M\left(G_{0}, G_{1}\right)$ is contained in an isometric 4-cycle. In particular, $P(10,3)$ admits no cubic contractions.

Proof: Suppose $e \in M\left(G_{0}, G_{1}\right)$. By the comment after Proposition 4.2 the graph $U_{0}=\left\{f_{0} \mid f \in\right.$ $\left.M\left(G_{0}, G_{1}\right)\right\}$ has degree one. If $f \in M\left(G_{0}, G_{1}\right)$ is such that $f_{0}$ is adjacent to $e_{0}$ then $e_{1}, e_{0}, f_{0}, f_{1}$ induce an isometric 4-cycle, as follows from Proposition 3.1.

Since the girth of $P(10,3)$ is six, this graph admits no cubic contractions.

For a cycle $C$ of a cubic graph $H$, an edge $e$ of $H$ is adjacent to $C$ at vertex $v \in C$ if $e$ is incident to $v$ and it does not lie on $C$. Recall that by the remark after Proposition 4.1, if an isometric cover $H_{0}, H_{1}$ defines a cubic expansion then $H_{0} \cap H_{1}$ is a disjoint union of edges.

Lemma 5.3 Suppose an isometric cover $H_{0}, H_{1}$ of a cubic graph $H$ defines a cubic expansion of $H$. Suppose $C$ is a cycle of $H$ that is convex as a subgraph of $H$. Suppose further that $e$ is an edge that is adjacent to $C$ at a vertex $v$. If $e$ is contained in $H_{0} \cap H_{1}$ then the following hold:

(1) $C$ has even length and $C \cap\left(H_{0} \cap H_{1}\right)=\{v, u\}$, where $u$ is the vertex opposite $v$ on $C$;

(2) one of the two halves of $C$ between $v$ and $u$ belongs to $H_{0}$ and the other one belongs to $H_{1}$;

(3) the edge adjacent to $C$ at $u$ lies in $H_{0} \cap H_{1}$. 


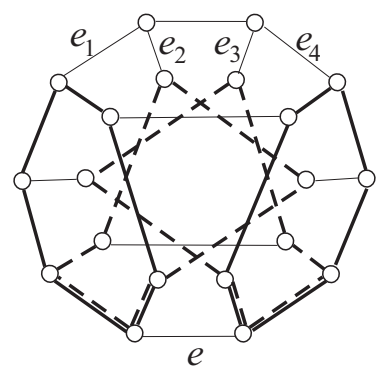

Fig. 2: Relation $\sim$ in $P(10,3)$.

Proof: Let $a$ and $b$ be the neighbors of $v$ on the cycle $C$. Since $e$ is contained in $H_{0} \cap H_{1}$, we have that both $a$ and $b$ do not lie in $H_{0} \cap H_{1}$. Without loss of generality, $a \in H_{0} \backslash\left(H_{0} \cap H_{1}\right)$ and $b \in H_{1} \backslash\left(H_{0} \cap H_{1}\right)$. Considering the path from $a$ to $b$ around $C$ (avoiding $v$ ), we see that $C$ contains another vertex $u$ that is contained in $H_{0} \cap H_{1}$. Since $H_{0}$ is isometric there is a shortest path between $u$ and $v$ that is fully contained in $H_{0}$. Since $C$ is convex, this path is contained in $C$. Similarly, there is a shortest path between $u$ and $v$ that is contained in $H_{1} \cap C$. Since $a \notin H_{1}$ and $b \notin H_{0}, C$ contains two different shortest paths between $u$ and $v$. This means that $C$ has even length and that $u$ is opposite $v$ on $C$.

This also means that such a vertex $u$ is unique. Therefore, the two edges on $u$, that lie on $C$, are not contained in $H_{0} \cap H_{1}$. It follows that the edge that is adjacent to $C$ at $u$ is in $H_{0} \cap H_{1}$.

When edges $e$ and $f$ of a graph $H$ are adjacent at opposite vertices to a convex cycle of even length, we will write $e \sim f$. This relation extends by reflexivity and transitivity to an equivalence relation on $E(H)$. This equivalence relation will be denoted by the same symbol $\sim$. It follows from Lemma 5.3 that if $H_{0}, H_{1}$ defines a cubic expansion of $H$ and $e$ lies in $H_{0} \cap H_{1}$ then the entire $\sim$-equivalence class of $e$ is contained in $H_{0} \cap H_{1}$.

Let us now see, what this gives for $P(10,3)$. Every 6-cycle in $P(10,3)$ is convex. Every edge $e$ is adjacent to four 6-cycles, cf. Fig. 2, where the four 6-cycles are emphasized.

Each of the 6-cycles leads to a new edge in the equivalence class of $e$, on Fig. 2 these are the edges $e_{1}, e_{2}, e_{3}$, and $e_{4}$. Continuing by transitivity, one establishes that all edges of $P(10,3)$ form a single $\sim$-equivalence class. This means that no cubic expansion is possible for $P(10,3)$. Combining this fact with Lemma 5.2 completes the proof of Proposition 5.1.

\section{Plane graphs}

The fourteen remaining examples from Table 1 are plane graphs with convex faces. (We say that a face of a plane graph is convex (respectively, isometric) if the boundary cycle of the face is a convex (respectively, isometric) subgraph.) These properties lead to a very explicit description of possible isometric covers and superconvex partitions and, as a result, to efficient algorithms for computing all cubic expansions and cubic contractions. We realized these algorithms in GAP (19) and used them for the tribe computations presented in the next section.

We also prove in this section that a graph, obtained by cubic expansion from a cubic plane graph with convex faces, inherits these same properties, so that our algorithms are again applicable. Cubic contractions preserve planarity, but not necessarily convexity of the faces. However, as we show in this 


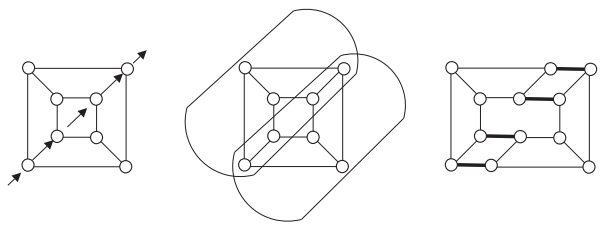

Fig. 3: A straight-ahead walk in $Q_{3}$, the corresponding isometric cover and cubic expansion.

section, non-convex faces can only arise in a very special situation that never occurs for our graphs.

Let $H$ be a plane cubic partial cube and $a b$ an edge of $H$. Let $W$ be the edge-face walk on $H$ that starts from $a b$ and continues as follows. Going along an edge $u v$ from $u$ to $v$, we enter the face at $v$ that is not incident with $u$. (The edge $u v$ is adjacent to this face at $v$.) In this face we proceed to the opposite vertex $v^{\prime}$ and exit the face along the edge adjacent to the face at $v^{\prime}$. We call $W$ a straight-ahead walk; see Fig. 3 where a straight-ahead walk in $Q_{3}$ is shown.

Proposition 6.1 Suppose $H$ is a plane cubic graph with convex faces. If $H_{0}, H_{1}$ form an isometric cover of $H$, defining a cubic expansion, then there exists a straight-ahead walk $W$ such that

(1) W has no self-intersections;

(2) for $i=0,1, H_{i}$ is induced by all vertices on one side of $W$ and on $W$ itself;

(3) $H_{0} \cap H_{1}$ is the disjoint union of the edges lying on $W$.

Suppose $H$ and $H_{0}, H_{1}$ are as above. For the proof of Proposition 6.1 we need the following simple fact to be used later as well and an important lemma classifying possible intersections of faces with $H_{0} \cap H_{1}$.

Proposition 6.2 For a graph $H$ and subgraphs $H_{0}$ and $H_{1}$, suppose $H=H_{0} \cup H_{1}$. Then $H_{0}, H_{1}$ is an isometric cover if and only if $d_{H_{0}}(u, v)=d_{H_{1}}(u, v)$ for all $u, v \in H_{0} \cap H_{1}$.

Lemma 6.3 Suppose $F$ is a face of $H$. Then one of the following holds:

(1) F fully lies in $H_{i}$ for some $i=0,1$, and meets $H_{0} \cap H_{1}$ trivially;

(2) F fully lies in $H_{i}$ for some $i=0,1$, and meets $H_{0} \cap H_{1}$ in an edge; or

(3) $F$ meets $H_{0} \cap H_{1}$ in two opposite vertices $v$ and $u$, the two edges adjacent to $F$ at $v$ and $u$ are contained in $H_{0} \cap H_{1}$, and the two halves of $F$ between $v$ and $u$ are contained in different $H_{i}$.

Proof: If $F$ meets $H_{0} \cap H_{1}$ trivially then, clearly, (1) holds. Suppose $v \in F \cap\left(H_{0} \cap H_{1}\right)$ and let $e$ be the unique edge on $F$ that is contained in $H_{0} \cap H_{1}$. If $e$ is adjacent to $F$ then (3) holds by Lemma 5.3. since $F$ is convex. It remains to consider the case where $e$ lies on $F$. We claim that in this case (2) holds. Suppose by way of contradiction that there is a third vertex $w$ in $F \cap\left(H_{0} \cap H_{1}\right)$. Let $f$ be the edge on $w$ that is contained in $H_{0} \cap H_{1}$. If $f$ is adjacent to $F$ then (3) must holds, a contradiction. Hence, $f$ lies on $F$. Taking, if necessary, the other end of $f$ as $w$, we can assume that $w$ is not opposite $v$. Since $F$ is convex and $v$ and $w$ are not opposite, there exists a unique shortest path between $v$ and $w$. However, by Proposition 6.2 this shortest path should be contained in both $H_{0}$ and $H_{1}$, a contradiction, since $H_{0} \cap H_{1}$ is a disjoint union of edges. 


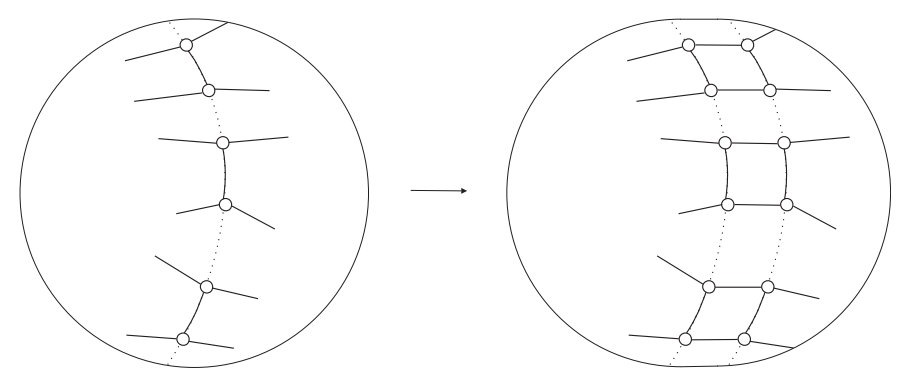

Fig. 4: Cubic expansion.

We are now ready to prove Proposition 6.1

Proof of Proposition 6.1. Suppose $e$ is an edge in $H_{0} \cap H_{1}$ and let $W$ be the corresponding straightahead walk. Since the faces of $H$ are convex, Lemma 5.3 implies that all edges on $W$ are contained in $H_{0} \cap H_{1}$. This shows that $H_{0} \cap H_{1}$ is a disjoint union of several straight-ahead walks. Suppose $W$ intersects another walk $W^{\prime}$ contained in $H_{0} \cap H_{1}$. (We allow for the equality $W^{\prime}=W$.) There are two possibilities for the intersection. If the intersection is at a vertex $w$ then each walk has an edge on $w$ (and this cannot be the same edge). This yields a contradiction, since the edges in $H_{0} \cap H_{1}$ are disjoint. If the intersection of $W$ and $W^{\prime}$ is at a face $F$ then Lemma 6.3 (3) gives a contradiction. Therefore, $H_{0} \cap H_{1}$ has no self-intersections.

If $H_{0} \cap H_{1}$ consists of more than one walk then cutting the sphere through all these walks splits the sphere into more than two parts. However, this means that $H_{0}$ and $H_{1}$ cannot be both connected. (Notice that this topological argument also can be used to show the absence of self-intersections.) Thus, $H_{0} \cap H_{1}=W$ is a single, non self-intersecting walk. This proves (1) and (3).

Considering the faces fully lying on one side of $W$, we see that either (1), or (2) of Lemma 6.3 must hold for each of these faces. Now connectivity implies (2).

Proposition 6.4 If $G$ is obtained by cubic expansion from a plane cubic graph with convex faces then $G$ is also a plane graph with convex faces.

Proof: Topologically, in this situation the cubic expansion amounts to cutting the plane graph $H$ along the walk $W=H_{0} \cap H_{1}$ and inserting a band with the new edges drawn across it, see Fig. 4 .

So $G$ has a natural structure of a plane graph. With respect to this structure, $G$ has three kinds of faces: (a) the faces of $H$, that are not affected by the expansion; (b) the faces affected by the expansion; these are the edges as in Lemma 6.3 (3); the gonality of these edges in $G$ increases by two; and (c) the new 4-gonal faces corresponding to the edges on $W$. Notice that the reverse operation of contraction transforms every path $\gamma^{\prime}$ on $G$ into a path $\gamma$ on $H$. The length of $\gamma$ is the length of $\gamma^{\prime}$ minus the number of times $\gamma^{\prime}$ crosses the inserted band.

Suppose $F^{\prime}$ is a face of $G$ and let $F$ be the corresponding face of $H$. (If $F^{\prime}$ is of type (c) then $F$ is an edge. Notice that an edge is convex as a subgraph of $H$.) Let $v^{\prime}$ and $u^{\prime}$ be vertices of $F^{\prime}$ and let $v$ and $u$ be the corresponding vertices of $F$. Consider a shortest path $\gamma^{\prime}$ in $G$ from $v^{\prime}$ to $u^{\prime}$ and let $\gamma$ be the corresponding path in $H$ from $v$ to $u$. Suppose first that $v^{\prime}$ and $u^{\prime}$ are on the same side of the band (that is, in the same $H_{i}^{\prime}$ for some $i=0,1$ ). If $\gamma^{\prime}$ crosses the band, then it crosses the band 
at least twice, so $d_{G}\left(v^{\prime}, u^{\prime}\right) \geq d_{H}(v, u)+2$. However, $d_{H}(v, u)=d_{H_{i}}(v, u)=d_{H_{i}^{\prime}}\left(v^{\prime}, u^{\prime}\right)$, and so $d_{G}\left(v^{\prime}, u^{\prime}\right)>d_{H_{i}^{\prime}}\left(v^{\prime}, u^{\prime}\right)$. This is a contradiction, since $H_{i}^{\prime}$ is a subgraph of $G$. Thus, $\gamma^{\prime}$ is fully contained in $H_{i}^{\prime}$. Now, since $H_{i}^{\prime}$ is a copy of $H_{i}$, it follows that $\gamma$ is a shortest path from $v$ to $u$ and so it is contained in $F$, since $F$ is convex. Hence also $\gamma^{\prime}$ is contained in $F^{\prime}$.

When $v^{\prime}$ and $u^{\prime}$ lie on different sides of the band, we similarly consider a shortest path $\gamma^{\prime}$ in $G$ and the corresponding $\gamma$ in $H$. In this case we show that the length of $\gamma$ is one less than the length of $\gamma^{\prime}$ and that again $\gamma$ is a shortest path from $v$ to $u$. It follows that $\gamma$ lies on $F$ and, consequently, $\gamma^{\prime}$ lies on $F^{\prime}$.

Next we turn to the operation of contraction. We start with a rather general lemma.

Lemma 6.5 Suppose $G_{0}, G_{1}$ form a superconvex partition of a graph $G$ and $C$ is an isometric cycle in $G$. Then one of the following holds:

(1) $C$ is fully contained in $G_{i}$ for some $i=0,1$; or

(2) $C$ has even length and it meets $M\left(G_{0}, G_{1}\right)$ in two opposite edges.

Proof: If none of the edges on $C$ is in $M\left(G_{0}, G_{1}\right)$ then case (1) holds. Suppose an edge $e$ from $C$ belongs to $M\left(G_{0}, G_{1}\right)$. Clearly, after crossing from one part of the partition to the other via $e, C$ must return back, so $C$ contains a second edge, say $f$, from $M\left(G_{0}, G_{1}\right)$. Since both parts $G_{i}$ are convex, $e_{1}$ and $f_{1}$ cannot lie on a shortest path between $e_{0}$ and $f_{0}$ and, vice versa, $e_{0}$ and $f_{0}$ cannot lie on a shortest path between $e_{1}$ and $f_{1}$. Since $C$ is isometric, this means that the part of $C$ between $e_{0}$ and $f_{0}$ is a shortest path between these two vertices and, similarly, the part of $C$ between $e_{1}$ and $f_{1}$ is a shortest path between them. Finally, Proposition 3.1 forces $C$ to have even length and $e$ and $f$ to be opposite.

We now return to the plane graphs. Suppose $G$ is such a graph and suppose that all faces of $G$ are isometric. Let $G_{0}, G_{1}$ form a superconvex partition of $G$. Since the faces are isometric, the above lemma implies that all faces, containing edges from $M\left(G_{0}, G_{1}\right)$, have even gonality and meet $M\left(G_{0}, G_{1}\right)$ in a pair of opposite edges. Let us make the edges from $M\left(G_{0}, G_{1}\right)$ the vertices of a new graph, in which two edges are adjacent whenever they are opposite each other on a face. Clearly, this is a graph of degree two and hence it is a union of cycles. Each of these cycles is called a zone (or, sometimes, a railroad). With each zone one can associate a cyclic zone walk on the sphere, in which $G$ is imbedded. Namely, starting from the middle of an edge we go across the face on one side of the edge to the middle of the opposite edge (which is again in the zone), cross into the next face, and so on until we return to the middle of the initial edge from the other side.

Proposition 6.6 Suppose $G$ is a plane graph with isometric faces and $G_{0}, G_{1}$ form a superconvex partition of $G$. Then $M\left(G_{0}, G_{1}\right)$ is a single zone. Furthermore, the corresponding zone walk has no selfintersections and each of $G_{0}$ and $G_{1}$ consists of all vertices on one side of the walk.

Proof: If a zone in $M\left(G_{0}, G_{1}\right)$ had a self-intersection or if there were two zones or more, then removing from $G$ all edges from $M\left(G_{0}, G_{1}\right)$ would result in more than two connected components, which is not the case. So $M\left(G_{0}, G_{1}\right)$ is a single zone and the corresponding walk has no self-intersections. The last claim also follows, since $G_{0}, G_{1}$ are the connected components of $G$ when all edges from $M\left(G_{0}, G_{1}\right)$ are removed.

Following the zone walk corresponding to the zone $M\left(G_{0}, G_{1}\right)$, let us write down the gonalities (the number of edges) of the faces that we cross. This gives a cyclic sequence $g_{1}, g_{2}, \ldots, g_{m}$, where $m$ is the number of edges/faces in the zone. We will call this sequence the zone sequence. 
Proposition 6.7 Suppose a plane graph $G$ is cubic with convex faces. Then the contraction defined by $G_{0}, G_{1}$ is a cubic contraction if and only if 4 appears in the zone sequence in every other place.

Proof: First of all, we argue that every 4-cycle in $G$ is a face. Indeed, suppose $C$ is a 4-cycle. Let $F$ be a face that contains two consecutive edges from $C$. Since $F$ is convex, we must have $F=C$.

Now for $e, f \in M\left(G_{0}, G_{1}\right)$, we have that $e_{0}$ and $f_{0}$ are adjacent if and only if $e_{0}, f_{0}, f_{1}, e_{1}$ is a 4-cycle (hence a 4-gonal face). So the claim follows from the remark after Proposition 4.2 .

Finally, we show that contractions preserve planarity and discuss under which conditions it preserves convexity of faces.

Proposition 6.8 If $G$ is a plane graph with isometric faces then the graph $H$ resulting from the contraction with respect to $G_{0}, G_{1}$ is again a plane graph with isometric faces.

Proof: By Proposition 6.6, $M\left(G_{0}, G_{1}\right)$ is a single zone and the corresponding walk has no self-intersections. This implies that the contraction amounts to cutting out a band from the sphere (cf. Fig. 4 with the reversed arrow) and gluing the two disks back together. So $H$ has a natural plane structure. Furthermore, the faces of $G$ that contain no edges from $M\left(G_{0}, G_{1}\right)$ retain their gonality in $H$, while the gonality of the faces containing edges from $M\left(G_{0}, G_{1}\right)$ goes down by two. The 4-gonal faces of this latter type disappear altogether.

Suppose $F^{\prime}$ is a face of $H$ and $F$ is the face of $G$ from which $F^{\prime}$ is produced. Suppose $v^{\prime}$ and $u^{\prime}$ are vertices of $F^{\prime}$, and suppose $\gamma^{\prime}$ is a shortest path between them. Let $G_{0}^{\prime}$ and $G_{1}^{\prime}$ be the images in $H$ of $G_{0}$ and $G_{1}$, respectively. Recall that each $G_{i}^{\prime}$ is isomorphic to $G_{i}$, and that $G_{0}^{\prime}, G_{1}^{\prime}$ form an isometric cover.

Suppose first that $v^{\prime}$ and $u^{\prime}$ are contained together in some $G_{i}^{\prime}$. Since $G_{i}^{\prime}$ is isometric in $H$, there is a shortest path between $v^{\prime}$ and $u^{\prime}$ that is fully contained in $G_{i}^{\prime}$. Let $v$ and $u$ be the preimages of $v^{\prime}$ and $u^{\prime}$ in $G_{i}$. Clearly, $v, u \in F$. We have $d_{H}\left(v^{\prime}, u^{\prime}\right)=d_{G_{i}^{\prime}}\left(v^{\prime}, u^{\prime}\right)=d_{G_{i}}(v, u)=d_{G}(v, u)$. Since $F$ is isometric, $d_{G}(v, u)=d_{F}(v, u)$. Also, $d_{F^{\prime}}\left(v^{\prime}, u^{\prime}\right) \leq d_{F}(v, u)$. This implies $d_{F^{\prime}}\left(v^{\prime}, u^{\prime}\right) \leq d_{H}\left(v^{\prime}, u^{\prime}\right)$, and since $F^{\prime}$ is a subgraph of $H$, the equality must hold.

Suppose now that $v^{\prime}$ and $u^{\prime}$ are in different $G_{i}^{\prime}$ 's. In particular, neither of the two vertices is in $G_{0}^{\prime} \cap G_{1}^{\prime}$. Without loss of generality we may assume that $v^{\prime} \in G_{0}^{\prime}$ and $u^{\prime} \in G_{1}^{\prime}$. Let $v$ and $u$ be the preimages of $v^{\prime}$ and $u^{\prime}$, respectively. We have that $v, u \in F$. Since every path from $v$ to $u$ necessarily contains an edge from $M\left(G_{0}, G_{1}\right)$, we must have $d_{H}\left(v^{\prime}, u^{\prime}\right) \leq d_{G}(v, u)-1$. To prove the reverse inequality, consider a shortest path $\gamma^{\prime}$ from $v^{\prime}$ to $u^{\prime}$. Let $x_{1}^{\prime}, x_{2}^{\prime}, \ldots, x_{k}^{\prime}$ be all the vertices from this path that are contained in $G_{0}^{\prime} \cap G_{1}^{\prime}$. The vertices on $\gamma^{\prime}$ that precede $x_{1}^{\prime}$ are in $G_{0}^{\prime}$. The vertices that follow $x_{k}^{\prime}$ are contained in $G_{1}^{\prime}$. Also, in view of Proposition 6.2 for every $i=1, \ldots, k-1$, we can substitute the subpath from $x_{i}^{\prime}$ to $x_{i+1}^{\prime}$ by a subpath of equal length, that is fully contained in $G_{0}^{\prime}$. Thus, without loss of generality, we may assume that all vertices preceding $x_{k}^{\prime}$ are in $G_{0}^{\prime}$. Now we can lift $\gamma^{\prime}$ to a path $\gamma$ in $G$ as follows: We lift the part of $\gamma^{\prime}$ from $v^{\prime}$ to $x_{k}^{\prime}$ into $G_{0}$, we lift the tail of $\gamma^{\prime}$, from $x_{k}^{\prime}$ to $u^{\prime}$, into $G_{1}$, and we connect the two parts of $\gamma$ by the edge from $M\left(G_{0}, G_{1}\right)$ between the two preimages of $x_{k}^{\prime}$. Clearly, $\gamma$ is a path from $v$ to $u$ and its length is one plus the length of $\gamma^{\prime}$. This shows that $d_{G}(v, u) \leq d_{H}\left(v^{\prime}, u^{\prime}\right)+1$. Thus, $d_{G}(v, u)=d_{H}\left(v^{\prime}, u^{\prime}\right)+1$.

Finally, since $d_{F}(v, u)=d_{F^{\prime}}\left(v^{\prime}, u^{\prime}\right)+1$ and since $d_{F}(v, u)=d_{G}(v, u)$, we get $d_{F^{\prime}}\left(v^{\prime}, u^{\prime}\right)=$ $d_{H}\left(v^{\prime}, u^{\prime}\right)$.

Unfortunately, it is not true that $F^{\prime}$ is convex whenever $F$ is. However, the conditions under which $F^{\prime}$ is not convex are rather restrictive and they do not occur in practice very often. 
Suppose $H$ is obtained from $G$ via a cubic contraction with respect to $G_{0}, G_{1}$. For $i=0,1$, let $G_{i}^{\prime}$ denote the image of $G_{i}$ in $H$.

Proposition 6.9 Under the above assumptions, let $F^{\prime}$ be a face of $H$ and $F$ be the corresponding face of $G$. Suppose further that $F$ is convex. Then $F^{\prime}$ is not convex if and only if it contains two or more edges from $G_{0}^{\prime} \cap G_{1}^{\prime}$.

Proof: Suppose the edges $e^{\prime}$ and $f^{\prime}$ from $G_{0}^{\prime} \cap G_{1}^{\prime}$ lie on $F^{\prime}$. Pick $v^{\prime}$ incident to $e^{\prime}$ and $u^{\prime}$ incident to $f^{\prime}$ so that $m=d_{H}\left(v^{\prime}, u^{\prime}\right)$ is minimal. Since $G_{0}^{\prime}$ is an isometric subgraph of $H$, there is a shortest path $\alpha^{\prime}$ from $v^{\prime}$ to $u^{\prime}$ that is fully contained in $G_{0}^{\prime}$. Similarly, there is a shortest path $\beta^{\prime}$ between $v^{\prime}$ and $u^{\prime}$ that is fully contained in $G_{1}^{\prime}$. Let $a^{\prime}$ (respectively, $b^{\prime}$ ) denote the vertex that follows $v^{\prime}$ on $\alpha^{\prime}$ (respectively, $\beta^{\prime}$ ). By the minimality of $m$, neither $a^{\prime}$, nor $b^{\prime}$ is incident with $e^{\prime}$. Since $e^{\prime}$ lies on $F^{\prime}$, we conclude that either $a^{\prime}$, or $b^{\prime}$ does not lie on $F^{\prime}$. Hence $F^{\prime}$ is not convex.

Now suppose $F^{\prime}$ is not convex, namely, there is a shortest path $\gamma^{\prime}$ between $v^{\prime}, u^{\prime} \in F^{\prime}$, that is not contained in $F^{\prime}$. We will assume that the length $m=d_{H}\left(v^{\prime}, u^{\prime}\right)$ of $\gamma^{\prime}$ is minimal among all such choices of $v^{\prime}, u^{\prime}$, and $\gamma^{\prime}$. In particular, none of the intermediate vertices from $\gamma^{\prime}$ lies on $F^{\prime}$. Note that this property holds for every path between $v^{\prime}$ and $u^{\prime}$ that has length $m$ and is not fully contained in $F^{\prime}$. Let $v, u \in F$ be preimages of $v^{\prime}$ and $u^{\prime}$. Let $i, j \in\{0,1\}$ be defined by $v \in G_{i}$ and $u \in G_{j}$.

Suppose some intermediate vertex $t^{\prime}$ from $\gamma^{\prime}$ is contained in $G_{0}^{\prime} \cap G_{1}^{\prime}$. Since $G_{0}^{\prime}$ and $G_{1}^{\prime}$ are isometric in $H$, we can assume without loss of generality that the part of $\gamma^{\prime}$ from $v^{\prime}$ to $t^{\prime}$ is fully contained in $G_{i}^{\prime}$ and the part from $t^{\prime}$ to $u^{\prime}$ is fully contained in $G_{j}^{\prime}$. Such a path can be lifted to a shortest path $\gamma$ from $v$ to $u$. (The length of $\gamma$ is $m$ if $i=j$, and it is $m+1$ if $i \neq j$.) Since $F$ is convex, we must have that $\gamma$ lies on $F$, and therefore, $\gamma^{\prime}$ lies on $F^{\prime}$, a contradiction. Thus, no intermediate vertex of $\gamma^{\prime}$ is in $G_{0}^{\prime} \cap G_{1}^{\prime}$. This means that all intermediate vertices are contained in $G_{k}^{\prime} \backslash\left(G_{0}^{\prime} \cap G_{1}^{\prime}\right)$ for some $k=0,1$.

If $i=k$ or $j=k$ then $\gamma^{\prime}$ can be lifted to a shortest path $\gamma$ from $v$ to $u$, again yielding a contradiction with convexity of $F$. Hence $i=j=1-k$. In particular, this implies that $v^{\prime}, u^{\prime} \in G_{0}^{\prime} \cap G_{1}^{\prime}$.

If $v^{\prime}$ and $u^{\prime}$ belong in the same connected component of $G_{0}^{\prime} \cap G_{1}^{\prime}$ (which is an edge!), then $m=1$, clearly giving a contradiction. Thus, $v^{\prime}$ and $u^{\prime}$ are incident to two different edges, say $e^{\prime}$ and $f^{\prime}$, from $G_{0}^{\prime} \cap G_{1}^{\prime}$. It remains to show that both $e^{\prime}$ and $f^{\prime}$ lie on $F^{\prime}$. However, this is clear. Indeed, by the minimality of $m$, the first edge along $\gamma^{\prime}$ does not lie on $F^{\prime}$. Hence the other two edges on $v^{\prime}$ must lie on $F^{\prime}$; one of them is $e^{\prime}$. By symmetry, also $f^{\prime}$ lies on $F^{\prime}$.

The condition for the non-convexity of $F^{\prime}$ can also be stated in terms of $G, F$ and the zone $Z=$ $M\left(G_{0}, G_{1}\right)$. Namely, $F^{\prime}$ is non-convex if and only if the zone walk corresponding to $Z$ crosses two different quadrangular faces adjacent to $F$ (but not $F$ itself).

We conclude this section with the following illustration of our theory.

Proposition 6.10 The even prisms $K_{2} \square C_{2 n}$ form a tribe.

Proof: It suffices to show that every cubic expansion of $K_{2} \square C_{2 n}$ coincides with $K_{2} \square C_{2 n+2}$ and that every cubic contraction of $K_{2} \square C_{2 n}$ coincides with $K_{2} \square C_{2 n-2}$.

We start with contractions. First of all, the cube $Q_{3}=K_{2} \square C_{4}$ has no cubic contractions, since it has no non-quadrangular faces. Let $n \geq 3$. In this case the prism has, up to symmetries, two types of edges: (1) the edges of the two $2 n$-gonal faces (top and bottom of the prism); and (2) the side edges, each of which is incident with two quadrangular faces (sides of the prism). The edges of the second kind form a zone. This zone does not lead to a cubic contraction because the zone sequence is the constant 4 . Each of 
the remaining zones looks the same: the zone walk goes across the top, then down the side, then across the bottom in the opposite direction, and then back up the opposite side. Manifestly, each of these zones defines a superconvex partition and the resulting graph is $K_{2} \square C_{2 n-2}$.

We now turn to expansions. When $n=2$, we have the cube $Q_{3}$, which has only one class of edges. Hence all straight-ahead walks are as in Fig. 3. Each of them defines an isometric cover and the resulting graph is the hexagonal prism. Now suppose $n \geq 3$. As above, we distinguish two kinds of edges. If a straight-ahead walk contains a side edge, then this walk goes up this edge, continues across the top, goes down the opposite side edge, and then crosses the bottom face back to the origin. Such straight-ahead walks do define isometric covers; indeed, the corresponding cubic expansions are the reverse operations to the cubic contractions from the preceding paragraph.

It remains to consider the straight-ahead walks containing the edges of the $2 n$-gonal top and bottom faces. Suppose, say, $e_{1}$ is an edge of the top face. The straight-ahead walk starting from $e_{1}$ goes diagonally down the next side face, then along an edge $f_{1}$ of the bottom face, climbs diagonally up the next side face, goes along the top edge $e_{2}$, and so on. If $n$ is odd, then the walk goes around the prism and eventually self-intersects. Suppose $n$ is even (and hence $n \geq 4$ ). In this case the straight-ahead walk has no selfintersections. However, it still does not define an isometric cover. Indeed, let $a$ be the second vertex on $e_{1}$ and let $b$ be the first vertex on $e_{2}$. (We count in the direction of the walk.) Let $H_{0}, H_{1}$ be the cover defined by the walk, where $H_{0}$ contains the top face and $H_{1}$ contains the bottom. Manifestly, $d_{H_{0}}(a, b)=3$, while $d_{H_{1}}(a, b)=5$. Thus, $H_{0}, H_{1}$ is not an isometric cover.

\section{Explicit tribes of partial cubes}

The theory that we have developed in the previous section leads to efficient algorithms listing all cubic expansions and contractions of a given plane cubic partial cube with convex faces. Suppose $H$ and $G$ are such graphs. To construct all cubic expansions of $H$, do the following:

(1) Construct all straight-ahead walks in $H$. Notice that each edge lies on a unique straight-ahead walk, so the straight-ahead walks partition the edge set of $H$.

(2) Select those straight-ahead walks $W$ that have no self-intersections.

(3) For each such $W$, construct $H_{0}$ and $H_{1}$ as subgraphs induces by the vertices on one side of $W$ and on $W$.

(4) Select isometric covers $H_{0}, H_{1}$ by verifying the distance condition as in Proposition 6.2 Notice that $H_{0} \cap H_{1}=W$.

To determine all cubic contractions of $G$, do the following:

(1) Construct all zones in $G$. Again, the zones partition the edge set of $G$, and so each edge needs to be dealt with only once.

(2) Select all zones $Z$, whose zone walks have no self-intersections and such that 4 appears in the zone sequence in every other place.

(3) For each such $Z$, construct $G_{0}$ and $G_{1}$ as the connected components of $G$ with all edges from $Z$ removed.

(4) Select superconvex partitions $G_{0}, G_{1}$ by verifying the distance condition as in Proposition 3.1.

In the case of cubic expansions, planarity of the resulting graph and convexity of its faces is assured by Proposition 6.4 Thus, the above algorithms can immediately be applied to construct further graphs from the same tribe. In the case of cubic contractions, planarity of the resulting graph is assured by Proposition 
6.8. while convexity of faces must be verified using Proposition 6.9 before the computation of the tribe can proceed.

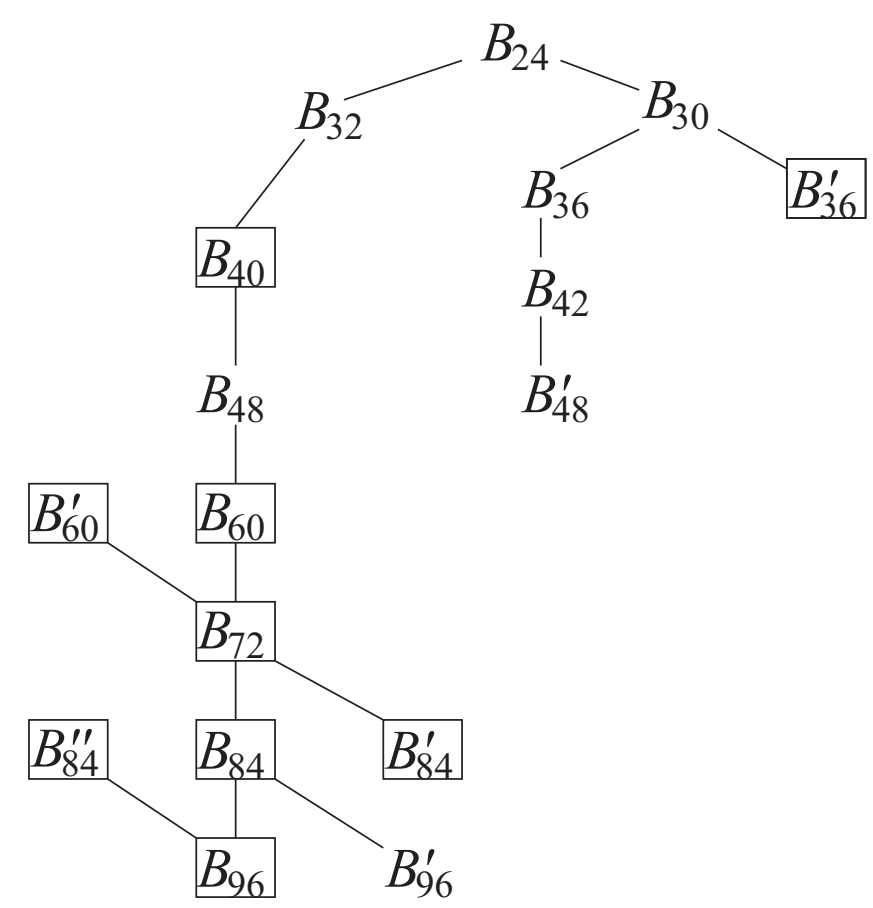

Fig. 5: The $B_{24}$-tribe.

We now present computational results based on the above approach. The first graph from Table 1 to consider is $B_{1}$. Set $B_{24}=B_{1}$ to denote the size of this graph. Then the $B_{24}$-tribe consists of 17 graphs and is schematically presented in Fig.5.

After the complete $B_{24}$-tribe has been determined, 9 new cubic partial cubes have been found. They are squared in the figure. The chain $B_{24}, B_{30}, B_{36}, B_{42}, B_{48}^{\prime}$ is the chain $B_{1}, B_{1}^{\prime}, B_{1}^{\prime \prime}, B_{1}^{\prime \prime \prime}, B_{2}$ from Table 1 . The graph $B_{32}$ is the chamfered cube, $B_{48}$ is the truncated cuboctahedron $\mathcal{C} \mathcal{I}\left(Q_{3}\right)$, while $B_{96}^{\prime}$ is $\mathcal{C} \mathcal{I}\left(G_{4}\right)$. The four new graphs with up to 60 vertices are depicted in Fig. 6

The next graph from Table 1 to consider is the twisted chamfered cube. Its tribe consists of just two graphs, the second graph from the tribe is the top graph from Fig. 7 It is not isomorphic to $B_{40}$, hence we have one more cubic partial cube. Similarly, the $\mathcal{C} \mathcal{I}\left(G_{1}\right)$-tribe consists of two graphs, the second graph has 60 vertices and is the middle graph from Fig. 7 Next, the $\mathcal{C} \mathcal{I}\left(G_{2}\right)$-tribe consists of three graphs, $\mathcal{C} \mathcal{I}\left(G_{2}\right)$ on 80 vertices and graphs on 70 and 60 vertices. So in this case both new graphs are obtained by a cubic contraction. The one with 60 vertices is the bottom graph from Fig.7.

Both the $\mathcal{C} \mathcal{I}\left(G_{3}\right)$-tribe and the $\mathcal{C} \mathcal{I}\left(G_{5}\right)$-tribe consist of a single graph. Finally, the $\mathcal{C} \mathcal{I}$ (Icosahedron)tribe is shown in Fig. 8, where we have set $I_{120}=\mathcal{C} \mathcal{I}$ (Icosahedron) to denote the size of this graph. The $I_{120}$-tribe thus brings us 8 new cubic partial cubes, the largest being on 240 vertices. It is the largest 

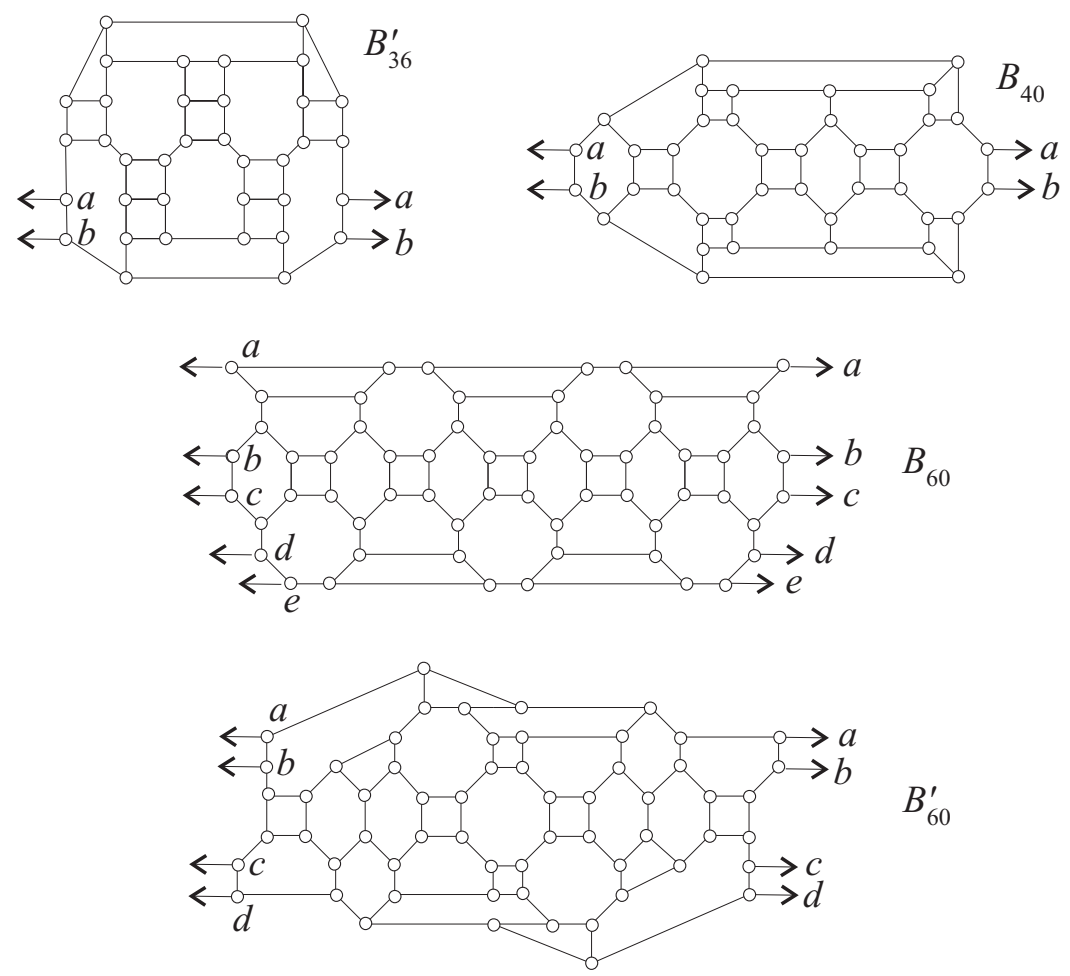

Fig. 6: Graphs from the $B_{24}$-tribe. 

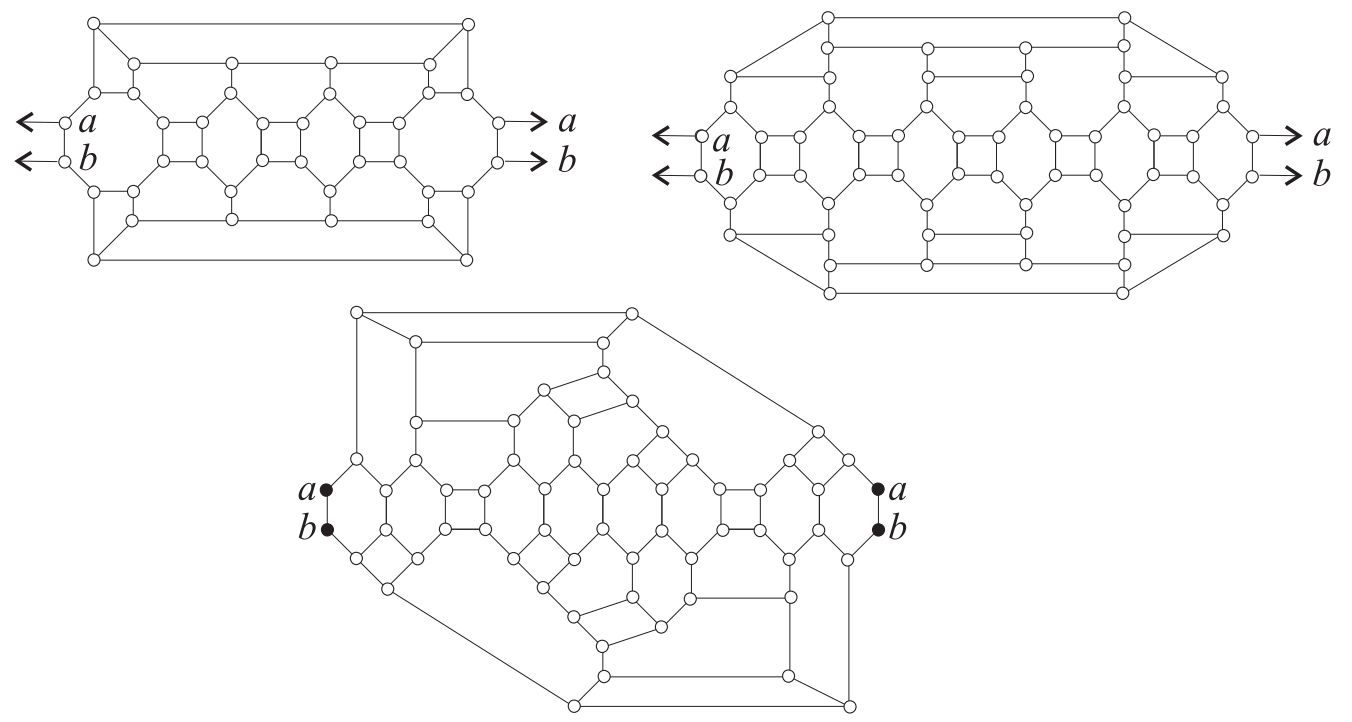

Fig. 7: The second graph from the twisted chamfered cube tribe, the second graph from the $\mathcal{C} \mathcal{I}\left(G_{1}\right)$-tribe, and the graph on 60 vertices from the $\mathcal{C} \mathcal{I}\left(G_{2}\right)$-tribe.

known cubic partial cube (that is not an even prism).

\section{Concluding remarks}

Among the known cubic partial cubes only the generalized Petersen graph $P(10,3)$ is not planar. Hence it would be interesting to find more nonplanar examples. It seems that for this sake new techniques need to be developed.

David Eppstein observed ((16)) that every simplicial line arrangement in the projective plane over the real numbers leads to a (plane) cubic partial cube. Three infinite series and 91 sporadic examples of simplicial arrangements are known (23). One of the three infinite series consists of the familiar prisms. It

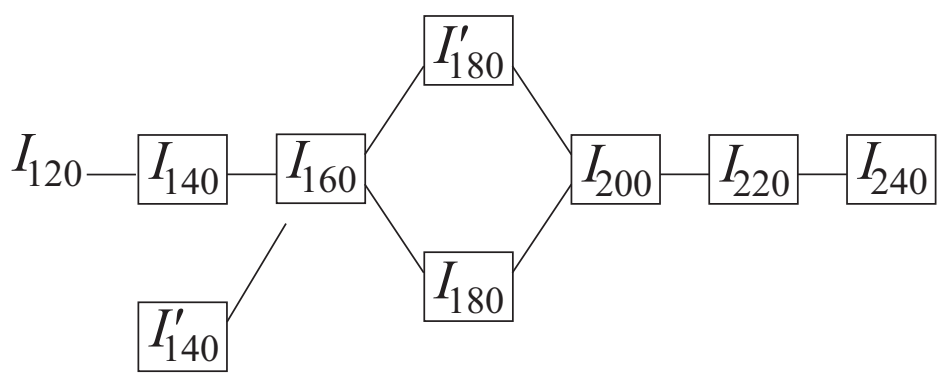

Fig. 8: The $I_{120}$-tribe. 
would be interesting to systematically consider all other examples to see to which known or new partial cubes they lead, and what their corresponding tribes are.

\section{References}

[1] D. Avis, Hypermetric spaces and the Hamming cone, Canad. J. Math. 33 (1981) 795-802.

[2] H.-J. Bandelt, M. van de Vel, Superextensions and the depth of median graphs, J. Combin. Theory Ser. A 57 (1991) 187-202.

[3] P. Bonnington, S. Klavžar, A. Lipovec, On cubic and edge-critical isometric subgraphs of hypercubes, Australas. J. Combin. 28 (2003) 217-224.

[4] B. Brešar, S. Klavžar, A. Lipovec, B. Mohar, Cubic inflation, mirror graphs, regular maps, and partial cubes, European J. Combin. 25 (2004) 55-64.

[5] D. Bryant, V. Moulton, Neighbor-net: An agglomerative method for the construction of phylogenetic networks, Mol. Biol. Evol. 21 (2004) 255-265.

[6] V. D. Chepoi, $d$-Convexity and isometric subgraphs of Hamming graphs, Cybernetics 1 (1988) 6-9.

[7] H. S. M. Coxeter, Regular Polytopes, Third Edition, Dover Publications, New York, 1973.

[8] M. Deza, M. Dutour-Sikiric, S. Shpectorov, Isometric embeddings of Archimedean Wythoff polytopes into hypercubes and half-cubes, MNF Lecture Notes Series, Kyushu University, Proc. Conf. on Sphere Packings (Fukuoka 2004), 55-70.

[9] M. Deza, M. Dutour-Sikiric, S. Shpectorov, Graphs $4_{n}$ that are isometrically embeddable in hypercubes, Southeast Asian Bull. Math. 29 (2005) 469-484.

[10] M. Deza, V. P. Grishukhin, A zoo of $l_{1}$-embeddable polytopal graphs, Bull. Inst. Math. Acad. Sinica 25 (1997) 181-231.

[11] M. Deza, V. Grishukhin, M. Shtogrin, Scale-Isometric Polytopal Graphs in Hypercubes and Cubic Lattices. Polytopes in Hypercubes and $\mathbb{Z}_{n}$, Imperial College Press, London, 2004.

[12] M. Deza, M. Laurent, Geometry of Cuts and Metrics, Springer-Verlag, Berlin, 1997.

[13] D. Djoković, Distance preserving subgraphs of hypercubes, J. Combin. Theory Ser. B 14 (1973) 263-267.

[14] A. Dress, M. Hendy, K. Huber, V. Moulton, On the number of vertices and edges in the Buneman graph, Ann. Combin. 1 (1997) 329-337.

[15] D. Eppstein, The lattice dimension of a graph, European J. Combin. 26 (2005) 585-592.

[16] D. Eppstein, Cubic partial cubes from simplicial arrangements, Electron. J. Combin. 13 (2006) \#R79, $14 \mathrm{pp}$.

[17] J.-Cl. Falmagne, S. Ovchinnikov, Media theory, Discrete Appl. Math. 121 (2002) 103-118. 
[18] K. Fukuda, K. Handa, Antipodal graphs and oriented matroids, Discrete Math. 111 (1993) 245-256.

[19] The GAP Group, GAP-Groups, Algorithms, and Programming, Version 4.4; 2005 (http://www.gapsystem.org)

[20] E. Gedeonova, Constructions of $S$-lattices, Order 7 (1990) 249-266.

[21] C. Godsil, G. Royle, Algebraic Graph Theory, Springer, New York, 2001.

[22] R. L. Graham, H. Pollak, On the addressing problem for loop switching, Bell System Tech. J. 50 (1971) 2495-2519.

[23] B. Grünbaum, Arrangements of hyperplanes, Proceedings of the Second Louisiana Conference on Combinatorics, Graph Theory and Computing (Louisiana State Univ., Baton Rouge, La., 1971), 41-106.

[24] B. Grünbaum, Arrangements and Spreads, American Mathematical Society, Providence, R.I., 1972.

[25] W. Imrich and S. Klavžar, Product Graphs: Structure and Recognition, Wiley, New York, 2000.

[26] W. Imrich, S. Klavžar, H. M. Mulder, Median graphs and triangle-free graphs, SIAM J. Discrete Math. 12 (1999) 111-118.

[27] S. Klavžar, Applications of isometric embeddings to chemical graphs, DIMACS Ser. Discr. Math. Theor. Comput. Sci. 51 (2000) 249-258.

[28] S. Klavžar, I. Gutman, B. Mohar, Labeling of benzenoid systems which reflects the vertex-distance relations, J. Chem. Inf. Comput. Sci. 35 (1995) 590-593.

[29] S. Klavžar, A. Lipovec, Partial cubes as subdivision graphs and as generalized Petersen graphs, Discrete Math. 263 (2003) 157-165.

[30] S. Klavžar, H. M. Mulder, Partial cubes and crossing graphs, SIAM J. Discrete Math. 15 (2002) $235-251$.

[31] B. Mohar, C. Thomassen, Graphs on Surfaces, Johns Hopkins University Press, Baltimore, 2001.

[32] H. M. Mulder, The structure of median graphs, Discrete Math. 24 (1978) 197-204.

[33] H. M. Mulder, The Interval Function of a Graph, Math. Centre Tracts 132, Mathemtatisch Centrum, Amsterdam, 1980.

[34] S. Ovchinnikov, Hyperplane arrangements in preference modelling, J. Math. Psychology 49 (2005) 481-488.

[35] P. M. Weichsel, Distance regular subgraphs of a cube, Discrete Math. 109 (1992) 297-306.

[36] E. Wilkeit, Isometric embeddings in Hamming graphs, J. Combin. Theory Ser. B 50 (1990) 179-197.

[37] P. Winkler, Isometric embeddings in products of complete graphs, Discrete Appl. Math. 7 (1984) 221-225. 
